\title{
The Suitability of Loctite 326 for Thermal Emission Measurements
}

\author{
M. C. Akin ${ }^{1} \cdot$ R. Chau ${ }^{1}$
}

Received: 19 July 2016/Accepted: 26 August 2016/Published online: 6 September 2016

(C) Society for Experimental Mechanics, Inc 2016

\begin{abstract}
The self-emissions of Loctite 326, Stycast 1266, and $\mathrm{LiF}$ were studied to determine their suitability for spectroscopy- and pyrometry-based shock temperature measurements. Stycast 1266 is anecdotally known to be a bright emitter; we observed significant non-thermal emission persisting over $400 \mathrm{~ns}$. Loctite 326 was found to have weaker emission at the same shock conditions, which depends on the degree of cure of the glue. Uncured Loctite emitted more brightly. LiF was found to assist cure of the Loctite and to provide an emission-free temporal window suitable for making temperature measurements. From this, we conclude that glues must be carefully considered, and ideally avoided, in shock experiments with $\mathrm{T}>2000 \mathrm{~K}$. If glues cannot be avoided, care must be given to the wavelengths used to determine $\mathrm{T}$, to avoid complications from non-thermal emission.
\end{abstract}

Keywords Pyrometry $\cdot$ Temperature $\cdot$ Shock physics . Equation of state

\section{Introduction}

Dynamic compression experiments are a key tool for equation of state measurements. The conservation of mass, momentum, and energy gives a simple yet powerful way to determine the pressure $(\mathrm{P})$, density, and total energy from a shock wave measurement [1]. However, temperature (T), an important state variable, is difficult to measure under

M. C. Akin

akin1@1lnl.gov

1 Lawrence Livermore National Laboratory, University of California, Livermore, CA 94550, USA shock loading. Short experimental timescales and high $\mathrm{T}$ require fast non-contact methods such as pyrometry and spectroscopy, where optical emission is measured.

To measure surface $\mathrm{T}$ at high $\mathrm{P}$, the surface must be tamped with a window; a free surface is necessarily at ambient $P$ [1]. Typical windows used in shock wave experiments are often single crystals that can have characteristic emissions or absorptions due to the formation of shear bands [2-4], impurities [5], and electronic phase transitions [6, 7]. However, the sample/window interface is imperfect. A simple joining of two materials leads to inevitable air gaps, which heat to $\mathrm{T}>11,000 \mathrm{~K}$ during shock loading, swamping out the sample's cooler gray body emission. Thus windows and other components are often attached with glues such as Loctite 326 or Stycast 1266.

These glues contribute their own thermal and non-thermal emissions. Here, we briefly communicate work to determine a sensible upper limit for using glue in our targets, and our observations of several notable pitfalls for glue use. The selfemissions of Loctite 326 and Stycast 1266 were studied to determine their suitability for spectroscopy- and pyrometrybased shock temperature measurements. Stycast 1266 is anecdotally known to be a bright emitter; we observed significant non-thermal emission persisting over $400 \mathrm{~ns}$. Loctite 326 was found to have weaker emission at the same shock conditions, which depends on the degree of cure of the glue. Uncured Loctite emitted more brightly. LiF was found to assist cure of the Loctite and to provide an emission-free temporal window suitable for making temperature measurements. From this, we conclude that glues must be carefully considered, and ideally avoided, in shock experiments with $\mathrm{T}>2000 \mathrm{~K}$. If glues cannot be avoided, care must be given to the wavelengths used to determine $T$, to avoid complications from non-thermal emission. 


\section{Methods}

We chose Loctite 326, as the optical emission has been cited as being very low versus other commonly used adhesives such as Stycast 1266, which is known to give a large emission signature [8]. LiF and silica were chosen as they are commonly used windows known to remain transparent in the pressures of interest.

Loctite 326 is a commercially available polyurethane methacrylate based single component adhesive, sold with a metal activator agent $(\mathrm{Cu})$ to facilitate curing. We do not use this agent as the results are too variable. Instead, we rely upon a metal interface, such as $\mathrm{Al}$, to cure the glue [9]. The $\mathrm{Li}$ of $\mathrm{LiF}$ appears to function well as this metal agent for thin layers typical of gas gun targets but is insufficient for thicker layers.

Based on our group's experience, we suspected degree of cure to be relevant in determining glue emission. To create a low-cure test target, we used fused silica and quartz. A test using Loctite 326 between two glass slides was found not to have cured or bonded even after a $72 \mathrm{~h}$ curing period. The failure to cure is attributed to the lack of metal ions in the quartz/fused silica-Loctite 326 combination.

Two LiF targets were built using degassed glues to examine high-cure response. Target One consisted of an $\mathrm{Al}$ baseplate $/ 2 \mu \mathrm{m}$ Stycast $1266 / 2 \mathrm{~mm} \quad \mathrm{LiF} / 5 \mu \mathrm{m}$ Loctite $326 / 2 \mathrm{~mm} \mathrm{LiF}$ sandwich. Target Two consisted of an Al baseplate $/ 3 \mathrm{~mm}$ Loctite $326 / 2 \mathrm{~mm} \mathrm{LiF}$ sandwich. Al was chosen for its low emissivity and excellent impedance match to LiF. While $3 \mathrm{~mm}$ Loctite 326 is unrealistically thick, it allows the emission of glue to be clearly measured as a way to estimate cure thickness. Two silica targets were assembled as well, consisting of fused $\mathrm{SiO}_{2} / 5 \mu \mathrm{m}$ Loctite 326/fused $\mathrm{SiO}_{2}$ sandwiches. A fused $\mathrm{SiO}_{2} / 3 \mathrm{~mm}$ Loctite 326/fused $\mathrm{SiO}_{2}$ sandwich was attempted, which showed no signs of curing/bonding and was not tested dynamically.

Seven-fiber bundles ( $400 \mu \mathrm{m}$ core fiber) were used to collect light and send it to six negatively biased photomultiplier tubes (PMTs) used for pyrometry measurements. As a result, an increase in emission appears as an increasingly negative voltage. Each channel had a band pass of $80 \mathrm{~nm}$ centered around 711, 611, (661 or 561), 511, 471 , and $413 \mathrm{~nm}$, and an appropriate neutral density filter to maintain signal in the linear region of PMT response. These wavelengths were chosen as we frequently use the visible region for measurements of $\mathrm{T}>2000 \mathrm{~K}$. Future work will extend this study to the infrared region used for measurements of cooler temperatures. The seventh channel was sent to a spectrometer (150 groove/mm grating) which was imaged by a Princeton Instruments PiMax II camera using a $2 \mathrm{~ns}$ exposure, to determine the overall shape of the emission and verify a lack of line emission. A two stage light gas gun fired copper impactors at velocities sufficient to compress the $\mathrm{LiF}$ to $73.5 \mathrm{GPa}$ in Target One and $88 \mathrm{GPa}$ in Target Two. Pressures in the fused $\mathrm{SiO}_{2}$ targets were roughly $28 \mathrm{GPa}$.

\section{Results}

Loctite was found to emit upon shock compression. However, the strength of this emission was found to depend upon cure levels; in the case of a thin layer of Loctite, which is well cured, very weak emission was observed (Fig. 1). This emission was seen only in the reddest two channels, indicating that the shock temperature of the glue layer is less than 1500-2000 K. While the red emission does continue, it acts as a small, flat offset to the initial emission levels until shock breakout from the LiF,

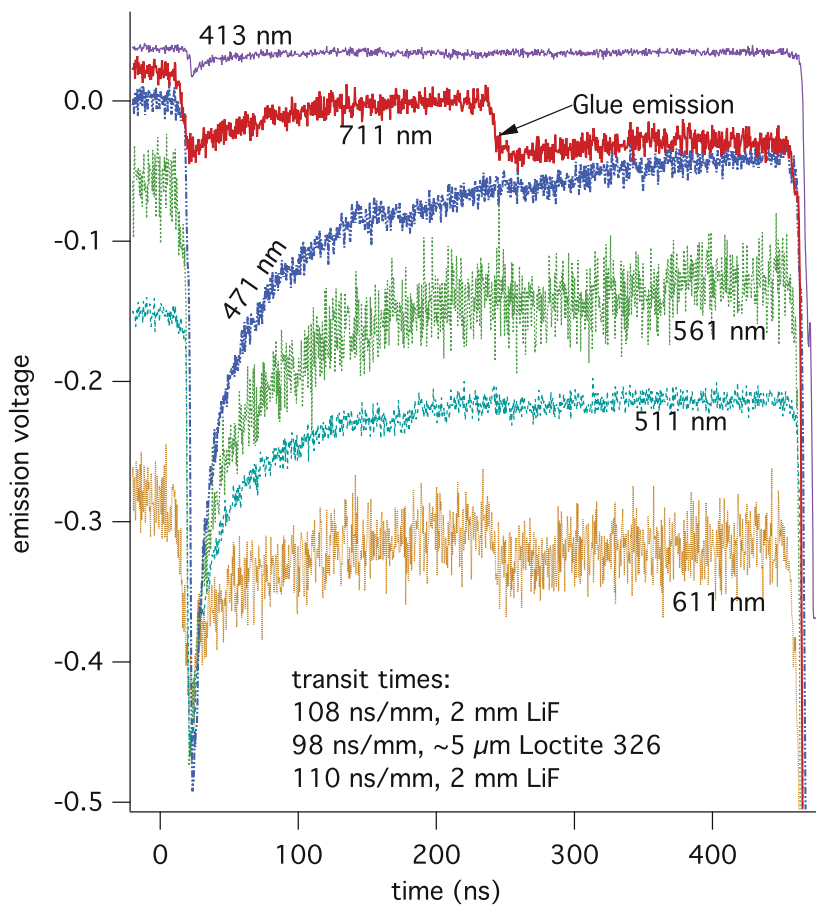

Fig. 1 (Color online) Shock emission from an Al baseplate/2 $\mu \mathrm{m}$ Stycast $1266 / 2 \mathrm{~mm} \mathrm{LiF} / 5 \mu \mathrm{m}$ Loctite $326 / 2 \mathrm{~mm} \mathrm{LiF}$ sandwich, shocked to $73.5 \mathrm{GPa}$ in $\mathrm{LiF}$. We see intense initial emission from the $\mathrm{Al} / \mathrm{Stycast} / \mathrm{LiF}$ interface, which decays smoothly but persists for hundreds of ns and appears to have a non-thermal component, with brighter emission in the blue region of the spectrum. The apparent persistent increase in emission at 711 and $611 \mathrm{~nm}$ occurring at $225 \mathrm{~ns}$ coincides with the arrival of the shock at the $\mathrm{LiF} / \mathrm{Loctite} / \mathrm{LiF}$ interface. The small signal change and lack of response in other channels suggest this emission is thermal; we roughly estimate $\mathrm{T}$ to be about $2000 \mathrm{~K}$. Based on this result, we use $73 \mathrm{GPa}$ in the glued LiF window as our everyday upper limit for Loctite in thermal targets, and use Stycast only when it is blocked optically by an opaque target component. Channels are offset for clarity 
and thus would not significantly alter temperature calculations. The flat nature of the additional emission indicates that no additional emission is contributed by the $\mathrm{LiF}$ window. In the fused $\mathrm{SiO}_{2}$ targets, the $\mathrm{SiO}_{2}$ is a much brighter emitter, such that the additional signal of the glue is not resolvable in the data.

In contrast, a thick layer of glue was found to emit significant amounts of light, as shown in Fig. 2. In this case, the glue emits for $141 \mathrm{~ns}$, which is much less than the transit time of the shock through the glue (about 210-300 ns, depending on model chosen). Close examination of the data prior to the identified onset of glue emission at $\mathrm{t}=72 \mathrm{~ns}$ in Fig. 2 shows only a very weak increase in signal equivalent to about $5 \%$ or less of the initial signal rise, beginning at about $16 \mathrm{~ns}$. We posit that this change in emission is due to changes in the degree of cure within the glue. The region of extremely low emission from 16 to $72 \mathrm{~ns}$ would correspond to the low emission seen from well-cured glue near the $\mathrm{Al}$ interface, with the region of high emission corresponding to poorly cured glue. This bright emission is largely thermal in nature, with no line emissions (Fig. 3). Of greater interest is the abrupt cessation of emission at $210 \mathrm{~ns}$ in Fig. 2. We know that this is when the shock front enters the $\mathrm{LiF}$ window as the predicted and observed transit times for $2 \mathrm{~mm} \mathrm{LiF}$ at this pressure are $204 \mathrm{~ns}$; shock breakout is observed at $414 \mathrm{~ns}$. From Fig. 2, we see that the LiF does not emit in any significant level, and that emission within the uncured glue layer occurs only during the initial shock. Approximately $60 \mathrm{~ns}$ after the shock enters the LiF, we see the onset of a

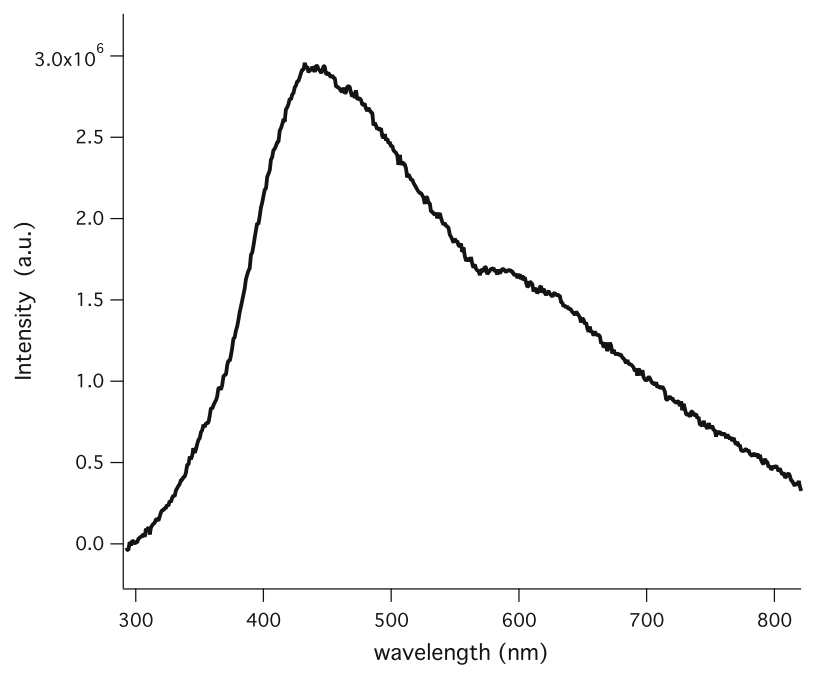

Fig. 3 Uncorrected self-emission from uncured region of $3 \mathrm{~mm}$ Loctite 326 as collected during a 2 ns exposure during shock transit, confirming that emission is largely thermal. As Loctite's composition is proprietary we cannot make further assignments

slow rise in total emission that appears to be thermal, which may be due to equilibration between glue and other layers.

\section{Discussion and Conclusions}

We demonstrate that the emission of thin (a few microns) Loctite 326 layers can be safely neglected below about $73 \mathrm{GPa}$ in the matching LiF window, even in relatively

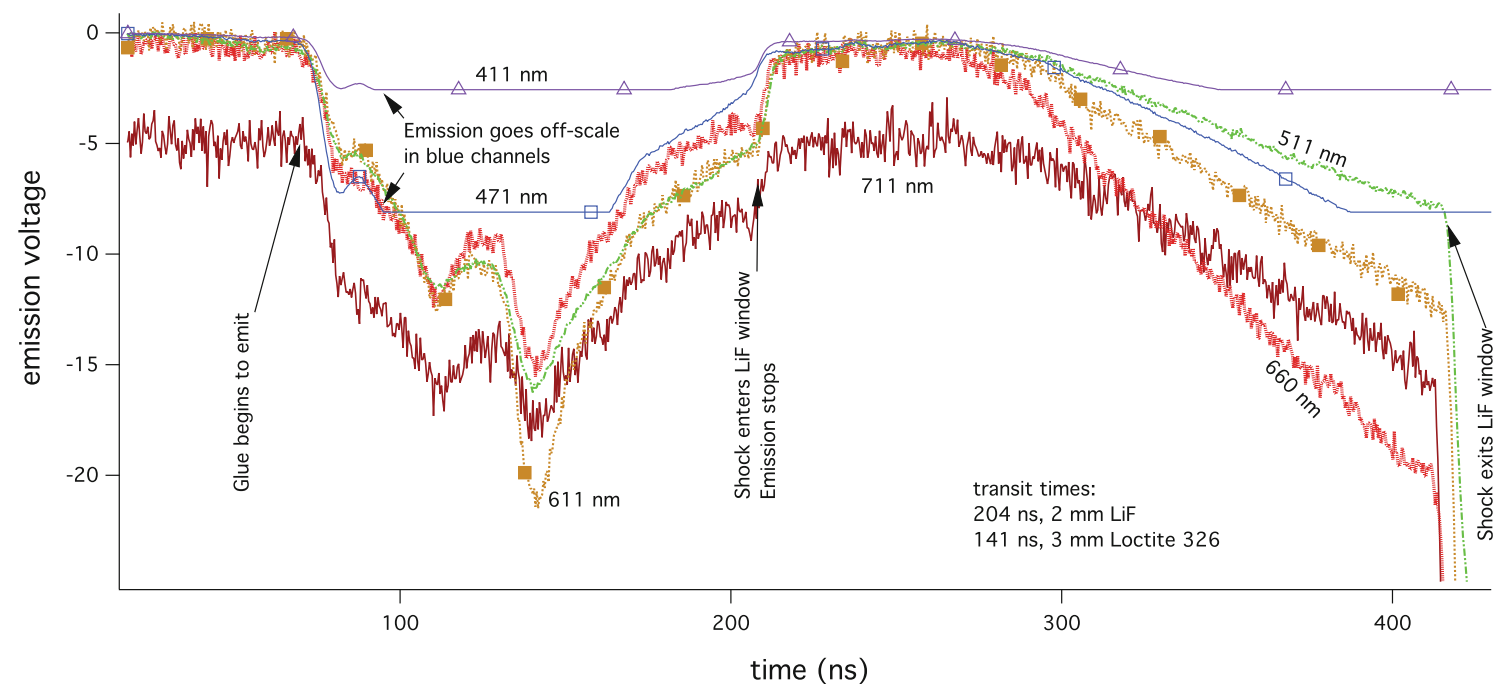

Fig. 2 (Color online) Shock emission from a $2 \mathrm{~mm} \mathrm{LiF} / 3 \mathrm{~mm}$ Loctite $326 / 2 \mathrm{~mm} \mathrm{LiF}$ sample, shocked to $88 \mathrm{GPa}$ in LiF. Selfemission of Loctite 326 is apparent for 141 ns during shock transit, but is quenched upon shock entry into the $\mathrm{LiF}$ window. If this bright emission begins when the shock enters the Loctite, then the shock speed is extremely high; it is more likely that the thick Loctite layer has varying degrees of cure and this emission began when the shock entered the uncured region. Re-emission begins after about $0.6 \mathrm{~mm}$ of LiF has been transited by the shock, followed by very bright emission as the shock breaks out of the LiF surface; the source of this reemission is unclear. The $711 \mathrm{~nm}$ channel is offset for clarity 
low-signal experiments, provided that the glue is fully cured. However, in cases where cure may be incomplete, emission from glue is much brighter, and is very likely to be a problem in later analysis. LiF was found to be a suitable window with minimal emission. Quartz and fused silica windows proved to be incompatible with this glue, failing to sufficiently cure and serving as bright emitters in their own right.

While these statements hold true for degassed Loctite 326 , the different chemistry between different glues, the method of cure, and possible gases produced during cure, may lead to dramatically different results with a different glue (as can be seen by the Stycast signal at $\sim 25 \mathrm{~ns}$ in Fig. 1). Even in the present case, the dramatic difference in the emission between the cured and uncured Loctite 326 suggests complicated shock induced organic chemistry that may be due to a differences in the bonding structure of the base polymers, and much greater heating in the still-liquid uncured glue. A study of such chemical reactions is beyond the scope of this work but shows the difficulty of using 'glues' in optically based dynamic compression experiments. With this in mind, we now use glue above the $73 \mathrm{GPa}$ threshold only with opaque foils or coatings in place to block any of its emission.

Acknowledgments We are thankful for the technical efforts of S. Caldwell, J. Streitz, S. Weaver, C. McLean, B. Nafzinger, and P. Benevento. This work performed under the auspices of the U.S.
Department of Energy by Lawrence Livermore National Laboratory under Contract DE-AC52-07NA27344. LLNL-JRNL-697787DRAFT.

\section{References}

1. Zel'dovich YB, Raizer YP (2002) Physics of shock waves and high-temperature hydrodynamic phenomena. Dover Publications Inc, Mineola

2. Hare DE, Holmes NC, Webb DJ (2002) Shock-wave-induced optical emission from sapphire in the stress range 12 to 45 gpa: Images and spectra. Phys Rev B 66(1):014108

3. Ogura T, Nakamura KG, Kondo K (2004) Time-resolved infrared radiometry of nacl crystals under shock compression between 17 and 43 gpa. Phys Rev B 70(14):144110

4. Schmitt DR, Ahrens TJ, Svendsen B (1988) Shock-induced melting and shear banding in single-crystal nacl. J Appl Phys 63:99

5. Kubicki JD, Hemley RJ, Hofmeister AM (1992) Raman and infrared study of pressure-induced structural-changes in mgsio3 camgsi2o6 and casio3 glasses. Am Mineral 77:258

6. Binggeli N, Troullier N, Martins JL, Chelikowsky JR (1991) Electronic-properties of alpha-quartz under pressure. Phys Rev B 44:4771

7. Stevens GD, Turley WD, Veeser LR, Jensen BJ, Rigg PA (2010) Reflectance changes during shock-induced phase transformations in metals. Review Sci Instrum 81(6):065101

8. Poulsen P, Baum D, Fiske P, Holtkamp D (2000) Temperature measurement on shocked surfaces. 51st Meeting of the Aeroballistic Range Association, Madrid, Spain, 18-21 Sept 2000

9. J. Jones (2011) personal communication 